\title{
Collaborative Approach Using Psychophysiology and Psychophysics for Optimal Threshold Determination in HAS Service QoE Management
}

\author{
Phan Xuan Tan, Eiji Kamioka \\ Graduate School of Engineering and Science, Shibaura Institute of Technology, Tokyo, Japan \\ Email: nb15507@shibaura-it.ac.jp,kamioka@shibaura-it.ac.jp
}

How to cite this paper: Tan, P.X. and Kamioka, E. (2018) Collaborative Approach Using Psychophysiology and Psychophysics for Optimal Threshold Determination in HAS Service QoE Management. Journal of Computer and Communications, 6, 57-81.

https://doi.org/10.4236/jcc.2018.68005

Received: July 18, 2018

Accepted: August 25, 2018

Published: August 28, 2018

Copyright $\odot 2018$ by authors and Scientific Research Publishing Inc. This work is licensed under the Creative Commons Attribution International License (CC BY 4.0).

http://creativecommons.org/licenses/by/4.0/

\begin{abstract}
In HTTP adaptive streaming (HAS) services, Quality of Experience (QoE) management has increasingly played an important role in guaranteeing the expected video quality perceived by the users with the minimal network resource. To achieve this, QoE must be automatically monitored and accurately controlled when it falls to a specific threshold. However, how to determine the QoE threshold with a scientific basis has not been carefully studied yet. In this paper, a new method to determine a QoE threshold based on biological information is proposed for a QoE management in HAS services. More concretely, the QoE threshold is determined by modeling the impact of the stimulus on the biological information obtained from the users who are watching videos. As the biological information, Skin Conductance and Heart Rate associated with Autonomic Nervous System (ANS) have been investigated. The evaluation results demonstrate that the QoE management by using the determined threshold can save more than $4.855 \%$ of the bandwidth consumption per control ensuring as high video quality as by using the fair threshold.
\end{abstract}

\section{Keywords}

QoE, HTTP Adaptive Streaming, Biological Information, Weber-Fechner Law

\section{Introduction}

Since HTTP Adaptive Streaming (HAS) technique was introduced in 2008, it has quickly become the primarily inspired approach for major online video providers [1]—including Akamai, Netflix, Apple, Adobe, etc. For years, HAS services 
have already reached a major market success. This is because HAS technique is based on a user-centric concept which is useful for designing the whole process of content production, service activation, content consumption and service management. According to [2], people around the world watch a billion hours of YouTube's video content every single day. In 2017, IBM [3] reported that two-thirds of adults subscribe to video on demand (SVOD) services according to a survey with more than one thousand US consumers. In addition, the SVOD users subscribe to more streaming services than ever before. These statistics bring not only a promising prospect but also a challenging issue to service providers, requiring a remarkable shift from technical Quality of Service (QoS) to perceived Quality of Experience (QoE). Therefore, QoE management has become a crucial issue for successful HAS services. There are numerous studies related to QoE management in HAS services [4] [5] [6]. In general, QoE management framework comprises of two major components, which are QoE monitoring and QoE control. QoE monitoring is responsible for observing network QoS and application QoS [7], and then for interpreting them to QoE in a real-time manner. On the other hand, QoE control is performed to recover the QoE level when it decreases under a specific threshold. In order to make an accurate decision for starting QoE control action, the QoE threshold as the trigger has to be seriously taken into consideration. However, the QoE threshold has not been carefully investigated before in literature.

Currently, different types of QoE assessement models for HAS services have been introduced. They can be classified into three types, namely, subjective models, objective models and hybrid models. The subjective models (or subjective assessment methods) refer to the process that quantifies the experienced quality of the user. In general, a panel of human is subjected to various quality levels which leads to some form of explicit or implicit response. However, the subjective assessment methods are costly, time-consuming and very complex to implement due to the involvement of human end users; thus, they cannot be applied to automatic and real-time in-service quality assessment [8]. In addition, according to [9] [10], rating approach which is recognized as the most common subjective assessment method, has significant drawbacks, mainly due to the high bias and variability. The objective models contain objectively collected measurements of QoE influence factors obtained from the environment where the user is watching a video. The motivation to use the objective models is that such the factors can be measured precisely with a lower cost than the subjective model. In addition, the objective models can be deployed on a wide range of systems in an automatic operation manner. The limitation of the objective models is that they often provide assessments that do not correlate well with human perception [11]. Furthermore, some of the objective models such as Peak Signal to Noise Ratio (PSNR) [12] and Structural Similarity Index (SSIM) [13] are based on computing a sort of distance between the reference video and the distorted video, that means, they need the reconstructed video, 
thus, they cannot be used in real-time. The hybrid models are the combination of subjective and objective models by leveraging the applications of machine learning techniques in order to model the relation between QoE influence factors and subjective assessment (or subjective evaluation). Afterward, the models can be used repeatedly in a real-time manner when it is needed [11]. Therefore, the hybrid models allow quantifying the experienced quality in a manner close to the human observation. As the result, in QoE management for HAS services, the hybrid QoE models are usually taken into consideration. As the most common hybrid model, Pseudo Subjective Quality Assessment (PSQA) [14] [15] was established by training a Random Neural Network (RNN) to capture the relation between QoS parameters and subjective evaluations by users. As the result, the trained network can be used for QoE estimation in automatic, accurate and real-time manners. The subjective evaluations are commonly performed by rating approach, where the users are asked to rate the quality of video content by 5 -scale Mean Opinion Score (MOS). Consequently, the fair level in 5-scale MOS (which is usually the middle value of the 5-scale) is simply selected as the threshold for QoE control [16] [17] [18]. However, as mentioned above, the rating approach are inheritably biased due to the qualitative nature of the scale (MOS scale) and present a high level of variance. As the result, the above threshold has to be re-determined. This leads to the demand of a new method to determine the optimal threshold for QoE control action.

Due to the limitation of rating by MOS, psychophysics approach has been considered in QoE assessment. This approach is applied to quantitative evaluations, clarifying the relation between a physical stimulus and a level of perception. Actually, such a relation was successfully modeled as a general logarithmic nature in Weber-Fechner Law, which has been applied in a number of QoE assessment studies [19] [20] [21] [22]. In this approach, the threshold level of stimuli which refers to absolute threshold is determined by introducing either a gradual increment until stimulus becomes detectable or a gradual decrement until stimulus becomes undetectable. In QoE assessment, such a threshold can be considered as the optimal one. Nonetheless, in psychophysics, still assessment scales like MOS and open ended questionnaires are typically used in order to quantitatively and qualitatively evaluate the user's perception to media content. These methods depend on conscious responses and often do not provide sufficient insight into underlying perceptual and cognitive process. Therefore, the psychophysics approach alone cannot provide necessary information for QoE assessment in HAS services.

In order to overcome the drawbacks of psychophysics, the approach of psychophysiology has been taken into account. The psychophysiology refers to physiological signals when stimuli are given, and then the correlations between the characteristics of the physiological signals and the given stimuli are discussed. The physiological measurements are categorized into the following classes [9]-Central Nervous System (CNS), Eye Measurements and Autonomic 
Nervous System (ANS). The psychophysiology detects the change of target stimulus through the change of corresponding physiological signal. It means that since the user's perception to the stimulus is obtained by the physiological signal, it achieves more precise QoE assessment. However, there is a significant limitation in the psychophysiology, which is the individual physiological difference that may produce systematic errors among subjects or groups thereof. Hence, a new approach which is applicable to a general population is required.

The abovementioned issues can be solved when the combination of the psychophysics and the psychophysiology is taken into consideration, compensating for the disadvantages of psychophysics with the advantages of psychophysiology, and vice versa. As the result, more precise QoE assessments without depending on individual physiological difference will be achieved. Accordingly, the optimal QoE threshold will be determined.

The research in this paper is to propose a new method to determine an optimal QoE threshold to ensure the perceived video quality stays at high and stable level with the minimal network resource utilization by taking into account the combination of psychophysiology and psychophysics in QoE assessment. The contributions of this research are as follows:

1) Establishing a general logarithmic function expressing the relation between human perception and stimulus intensity. Based on the function, the human perception is estimated through physiological measurements (Note that in this paper, the term of "biological information" will be used instead of "physiological information"), and stimulus intensity will be referred to as a QoE influence factor.

2) Defining an optimal QoE threshold by determining the absolute threshold or its constraint.

This paper is organized as follows: Section 2 will describe the related work followed by the review of research background in Section 3. The proposal in this research will be introduced in Section 4, including the hypotheses description and the analytical model. Section 5 will show the evaluation experiment and the numerical results. The discussion of the evaluation will be presented in Section 6 . Finally, the conclusion and future directions will be stated in Section 7.

\section{Related Work}

QoE control is one of the indispensable components in QoE management. Its mechanism is quite simple. When QoE level falls under a specific threshold, the control component will trigger a certain action (e.g. bandwidth re-allocation to the user, performing admission control and so forth) to recover the QoE to the expected level. The operation of QoE control is validated when the precise control action is generated at the right time. To achieve this, defining a control threshold plays an important role. If the threshold is set to a higher value than the actual one, the network resource utilization cannot be optimized. If the threshold is set to a lower value than the actual one, the QoE deterioration 
cannot be detected early enough, and thus, the user will have already perceived the deterioration when QoE control is performed. As mentioned previously, in order to determine an optimal QoE threshold, the combination of psychophysiology and psychophysics in QoE assessment need to be studied.

Psychophysiology has been increasingly applied in QoE assessment for multimedia services, including HAS services [9]. There are numerous studies which focus on QoE assessment by using biological measurements. [23] shows a significant correlation between Electroencephalography (EEG)/Electrocardiography (ECG) and video quality levels, [24] presents that Electrodermal Activity (EDA) is effective to measure the perception to given visual fatigue, whereas [25] concludes that the same result was found from both EEG and EDA. Particularly, in the effort to directly measure the perceived video quality changes using EEG, the authors in [26] concluded that abrupt changes of video quality give rise to specific components in the EEG that can be detected in a single-trial basic. Potentially, a neurotechnological approach to video assessment can lead to more objective quantifications of quality change detection, overcoming the limitation of subjective approaches (such as subjective bias and the requirement of overt response). Those contributions promisingly motivate applying psychophysiology in QoE management for HAS services. In this research, the biological information (EDA and HR) associated with Autonomic Nervous System (ANS) is initially used to determine the appropriate QoE threshold.

Estimating human's perception using the intensity of a specific stimulus has attracted many scientists for years. The initial cornerstone was marked by the breakthroughs of Weber and Fechner. The relation between human perception and stimulus intensity was modeled as a logarithmic nature expressed by Weber-Fechner Law. Consequently, the Weber-Fechner Law has been widely applied in many research areas [19] [20] [27] [22]. In this research, the optimal QoE threshold is determined by modeling the impact of stimulus intensity on human perception based on the logarithmic nature.

\section{Background}

Section 2 clarifies the applications of biological measurements in QoE assessment, especially ECG and EDA which are associated with ANS. Considering the efficiency and the convenience for the users, EDA and heart rate (HR) were eventually selected as measured signals in this research. This section investigates the background knowledge related to the terms of biological signals (EDA and HR), and psychophysics (Sensation, Perception and Weber-Fechner Law).

\subsection{Electrodermal Activity}

In human's perceptual process, sensory receptors convert sensations into electrical impulses [29]. The electrical impulses are relayed to the brain and the responses to the stimulations are induced. Electrodermal activity refers to changes in electrical conductance of the skin, which is associated with eccrine sweat gland 
activity innervated by the sympathetic branch of the autonomic nervous system. Measuring electrodermal activity (EDA) is a promising way to determine the amount of an individual's response to stimuli [30] [31]. Thus, it can be a useful index of changes in sympathetic arousal that is tractable to emotional and cognitive states. Skin conductance (SC) is the most widely studied property of EDA, representing autonomic changes in the electrical properties of the skin. SC comprises of two major components-tonic and phasic. Tonic component is represented by skin conductance level (SCL) which is the baseline level of SC, in the absence of any particular discrete environmental event. On the other hand, phasic component refers to skin conductance responses (SCRs) reflecting the short-time response to a given stimulus. SCRs are also the results from sympathetic neuronal activity.

In SC qualitative modelling or poral valve modelling [32], SC is the result of a numerous activities related to sweat ducts, pore, sweat glands, etc. In the initial condition, the distal part of the sweat ducts is collapsed by the external pressure of hydrated surrounding corneum. As the result, most of the pores are closed. When the sweat fills the ducts to their limitation of capacity, the intraductal pressure will cause a hydraulic driven diffusion of sweat into the corneum. The rising of SC is caused by the increasing the hydration in deeper levels of the corneum. As the sweat is reabsorbed into the dermis or diffuses away from the periductal area, SC will slowly recover, resulting in a rather flat SCR. When the secretion of the sweat is enough and the intraductal pressure becomes stronger than the tissue pressure of the corneum, the pore will eventually open. The sweat will now be forced out through the pore. Consequently, SC will drastically increase. The amount of sweat which is pushed out through the pore is substantial. Therefore, after a short time, the secretory rate cannot keep up with the loss of sweat. The intraductal pressure will soon fall below the tissue pressure, and the pores will be collapsed again followed by a rapid fall in SC. Basically, SCR shape can primarily be ascribed to two different underlying processes: one is an unconditional diffusion process which causes a rather flat SCR, and the other is an optional opening of pores which will add a steep peak to the basic SCR shape. Thus, these processes increase the variability of the SCR shape. Figure 1 depicts an example of SCR shape which comprises the following components-Latency (time from stimulus onset to SCR onset), Rise time (time from SCR onset to SCR peak) and Half recovery time (time from SCR peak to 50\% recovery of SCR amplitude). In the figure, the vertical and horizontal axes represent skin conductance value and duration time, respectively.

\subsection{Heart Rate}

The heart rate (HR) is rhythmically generated by the sinoatrial node. It is a reliable reflection of the many biological factors modulating the normal rhythm of the heart. In fact, it provides a powerful means of observing the interplay 


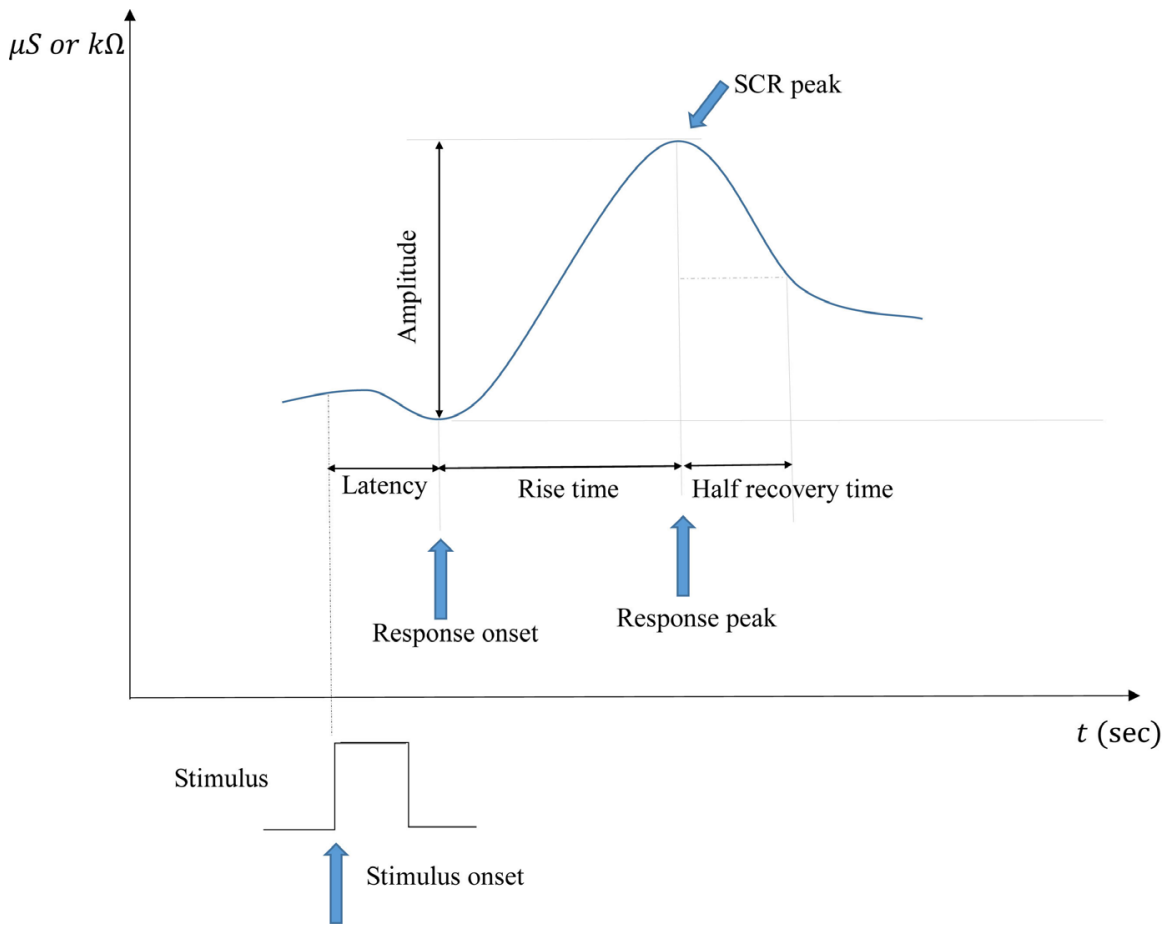

Figure 1. The graphical representation of principal SCR related components [6].

between the sympathetic and parasympathetic nervous systems. HR is a useful factor for understanding the status of ANS due to the non-stationary signal. The sympathetic stimulation causes an increase in HR by increasing the firing rate of pace-maker cells in the heart's sino-atrial node. Whereas, parasympathetic activity decreases the firing rate of pace-maker cells and the HR, providing a regulatory balance in physiological autonomic function. The balancing action of the sympathetic nervous system and parasympathetic nervous system controls the HR. The degree of variability in the HR provides information about functioning of the nervous control on the HR and the heart's ability to respond. Thus, investigating the behavior of HR can express the impact of stimulus intensity on human reaction. In addition, HR measurement has becomes easier than ever due to the abundance and diversity of monitor devices as well as the convenience in measurement process.

\subsection{Sensation, Perception, Weber Law, and Fechner Laws}

Although the terms of sensation and perception are often used interchangeably, it is important to clarify the difference between them. The sensation is the process in which the sensory receptors and nervous system receive stimulus energy from the outside environment. It also represents the amount of the stimulus energy. Meanwhile, the perception is the process of organizing and interpreting sensory information, enabling human to recognize meaningful objects and events. More concretely, the sensory process captures information from the outside world and transforms it into biological signals that are interpreted by the brain [33] [34]. Afterward, the brain produces a perceptual 
representation that allows human to appreciate the outside world. In other words, the perception represents a single unified awareness of a stimulus that in turn arises from the sensation produced by the sensory system.

As mentioned in Section 1, human perception is an important role in QoE assessment. Typically, the good way to understand this concept is to establish a quantifiable relation between the physical stimulus and the perception. Consequently, the perceptual quality of a stimulus can be represented in numerical terms, enabling the comparison with other stimuli [33]. Weber and Fechner were experimental psychologists of the 19th century who made efforts in establishing such a relation. Before going further, the definitions of both "absolute threshold" and "difference threshold" which are of crucial in this research, need to be clarified.

As mentioned in [33] [34] [35] [36], the absolute threshold is defined as the minimum level of stimulus intensity that can be recognized as a sensory event by the brain. The stimulus intensity in the range of lower than the absolute threshold is called as subthreshold intensity. No detectable sensation is produced from subthreshold intensity. On the other hand, the stimulus intensity higher than the absolute threshold is called supra-threshold intensity. Sensation takes place from the supra-threshold. In the supra-threshold intensity, a certain level of intensity change either increment or decrement intensity can be detected as perception. The difference threshold concept came up to determine how much change in stimulus intensity is needed to recognize the difference in sensation, which is called Just Noticeable Difference (JND).

Fechner tried to understand the relation between stimulus intensity and perception. To achieve this, the absolute threshold was determined by a series of experimental approach. In this experiment, the reference stimulus with a constant intensity was prepared. Besides the reference stimulus, the subjects were given different stimulus with intensity randomly varied in pre-defined range. They compared each given stimulus with the reference stimulus and answered the "Yes" or "No" which corresponds to whether the stimulus change was perceived or not. As illustrated in Figure 2, the absolute threshold is determined as a stimulus intensity with which the stimulus was perceived by the $50 \%$ of the subjects. Whereas, the intensities at which the $25 \%$ and $75 \%$ of "yes" response were obtained are considered as just noticeable intensities for incremental and decremental sensation, respectively, according to the definition of JND.

The Weber's Law answers the question how the absolute threshold, just noticeable intensity and JND change when the reference intensity varies. After conducting the series of experiments, Weber found that the difference threshold increases in a linear fashion with stimulus intensity as shown in Equation (1) and Figure 3. It means that higher supra-threshold intensity requires larger change in intensity $(\Delta I)$ needed to produce a change in sensation, namely, JND.

$\Delta I=k * I$ 


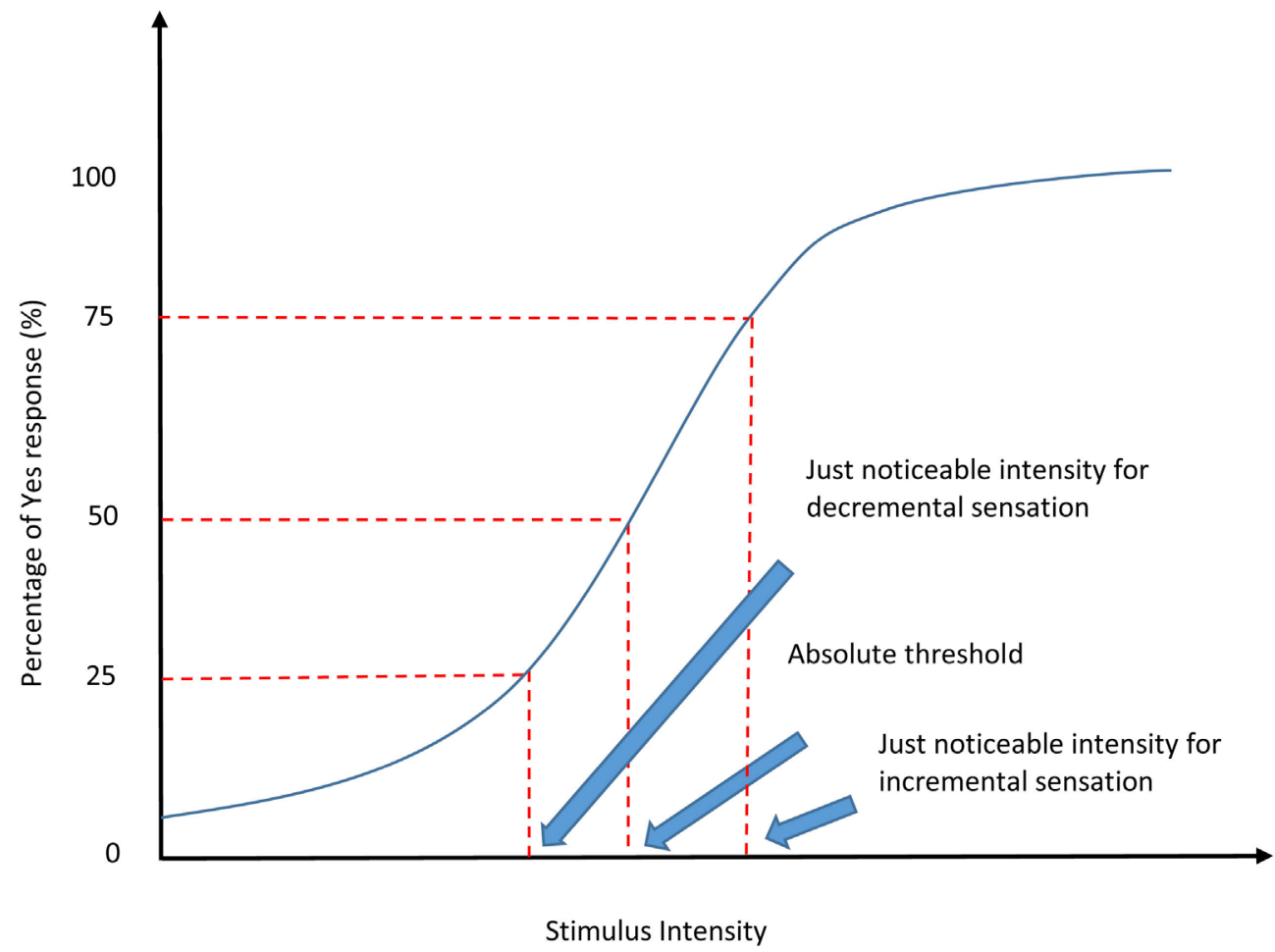

Figure 2. Absolute threshold obtained from psychometric function.

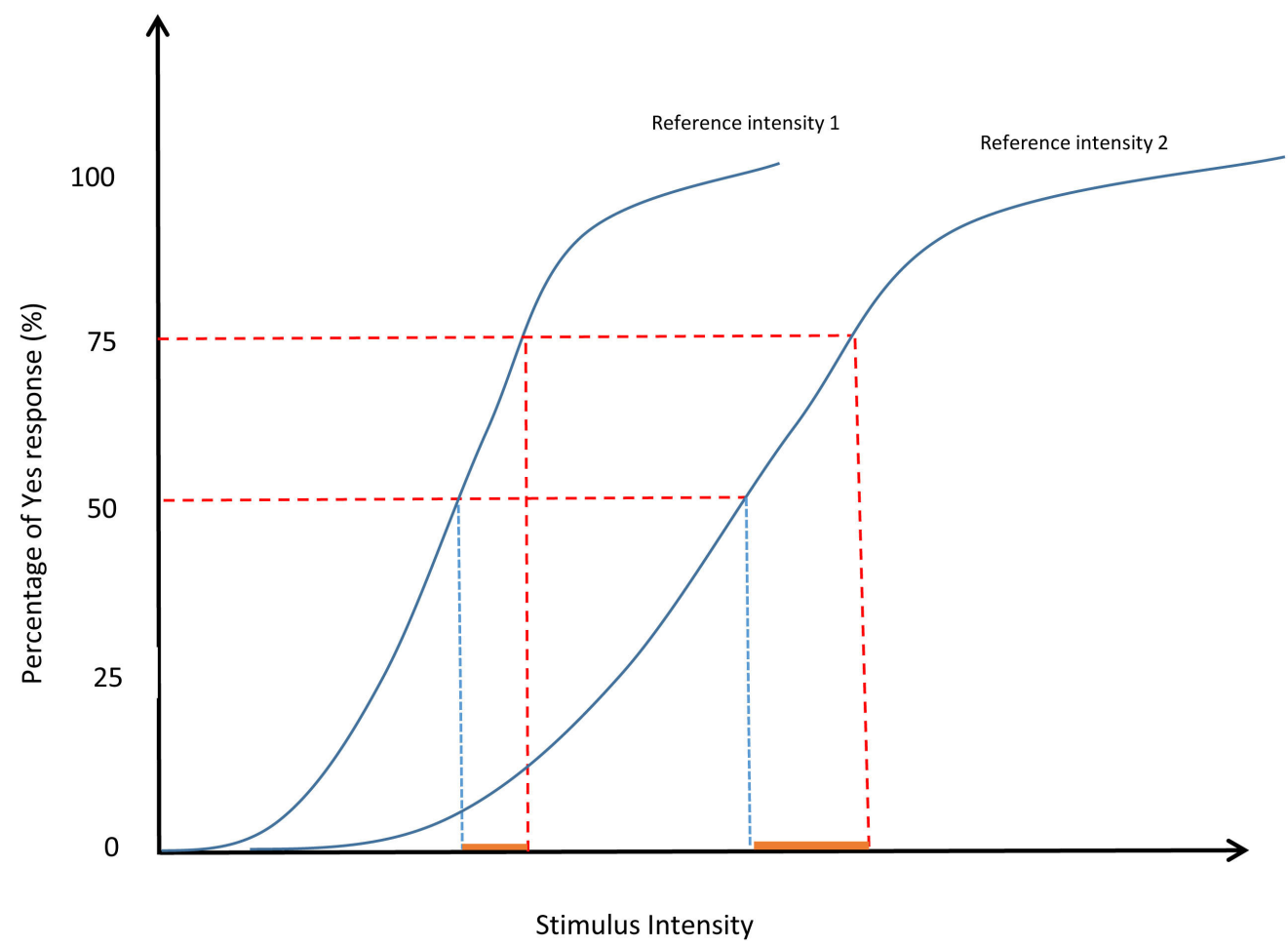

Figure 3. The greater change in absolute threshold, just noticeable intensity when the reference intensity progressively increases.

After the introduction of the Weber's Law, Fechner eventually figured out the relations between stimulus intensity and perception by integrating his bold 
assumption with Weber's Law. The assumption was stated as: the subjective size of the JNDs to be constant, irrespective of sensation magnitude. This assumption can be mathematically achieved in a logarithmic function. The Equation (2) completely expresses his idea:

$$
S=k^{\prime} \log I
$$

where constant $k^{\prime}$ is related to, but not identical to, the constant $k$ in Weber's Law. $S$ is the magnitude of sensation, whereas $I$ stands for stimulus intensity, and $\log I$ indicates natural $\operatorname{logarithm}$ of $I$. It can be concluded that both data points whose intensities are equal to the absolute threshold and the just noticeable intensity must belong to the curve of Equation (2).

\section{Methodology}

In this section, the hypotheses in this research are introduced in detail, explaining how they connect to the proposal.

\subsection{Hypotheses}

Looking back to Section 1, in this research, a new method to ascertain the optimal QoE threshold for control component in QoE management is proposed. More concretely, the collaborative approach using the psychophysiology and the psychophysics to clarify a general logarithmic nature function between human perception and stimulus intensity is thoroughly discussed. In this research, the perception has been estimated via biological information (including SC and HR) measurements. Afterward, either absolute threshold or just noticeable intensity is expectedly obtained from the general logarithmic nature function, which facilitates the determination of optimal QoE threshold.

In order to achieve this, the following hypotheses must be justified:

- Biological reaction to a stimulus change varies from person to person and depends on the types of biological information. It can be detected by an increment or decrement of the amplitude.

- Biological information, which shows the level of human perception to stimulus intensity, has a logarithmic nature relation with the stimulus intensity.

- The absolute threshold which is the minimum stimulus intensity perceived by $50 \%$ of subjects is ideally regarded as the stimulus threshold.

\subsection{Modelling of Biological Information}

In order to model a general logarithmic nature between human perception and stimulus intensity, initially, the type of stimulus given to the human needs to be clarified. This can be done by investigating the prominent QoE influence factors. According to [37], there are numerous influence factors categorized into perceptual and technical factors. Video rate in HAS services influence the quality of video frame, hence, it can be regarded as one of the factors directly perceived by subjects. Therefore, it was chosen as the stimulus type in this research. There 
are copious existing models which have a capability to interpret the video rate to QoE (indicated by Mean Opinion Score) and vice versa [38] [39], and thus, the video rate threshold can be used for the trigger of control action in QoE management, instead of QoE threshold.

In Section 2, the wide range of Weber-Fechner Law (WFL) applications has been introduced, especially in QoE assessment throughout modelling the relation between QoE and QoS. However, subjective QoE is insufficient to characterize the human perception in QoE assessment. On the other hand, the human perception introduced by biological information promisingly tells us the truth on how stimulus intensity is perceived. Additionally, since more precise perception can be derived from the combination of different types of biological information [9], it is necessary to jointly investigate the impact of stimulus on multiple biological signals. In this subsection, by applying WFL, the impact of the video rate on the human perception estimated by multiple biological information, which are Skin conductance (SC) and Heart rate (HR), is modeled. Therefore, the Equation (2) in Subsection 3.3 can be re-written as follows:

$$
y_{i}=k_{i}^{\prime} \log x
$$

where $i \in\{1,2, \cdots, n\}$ is the index indicating each subject, and $y_{i}$ is the perception level of subject $i$ to the stimulus of SC or HR, respectively. Let $k_{i}^{\prime}$ be the sense-specific constant depending on the sense (SC and HR) and type of stimuli.

In order to achieve the research purpose, the following tasks need to be covered:

- Establish the collection of $n$ regression curves of Equation (3) by fitting data obtained from each subject.

- Investigate the existence of the data point which is crossed by at least $50 \%$ of regression cruves.

- Determine the general logarithmic function representing for all subjects from the data point which is determined in the second step.

- Determine the absolute threshold.

To clarify the above tasks, more explanations are needed. As mentioned in hypotheses, the perception varies from person to person and depends on the type of stimulus, thus, the shape of regression curve of Equation (3) is predictably different in each subject, only meaning that each subject separately perceives the stimulus intensity. If $50 \%$ and more than $75 \%$ of subjects have the same perception magnitude to the same intensities, they are considered as the absolute threshold and the just noticeable intensity, respectively. Then, those regression curves are expected to intersect at the same data point denoted by $P\left(x_{0}, y_{0}\right)$. According to the conclusion of Subsection 3.3, if this data point exists, it must be the crossed point by the curve of the general logarithmic functions $y$. Data point $P\left(x_{0}, y_{0}\right)$ can be determined by minimizing the sum of squared residuals, defined as the square of the difference between $y$ and $y_{i}$ : 


$$
f(x, y)=\sum_{i=1}^{\infty}\left(y-y_{i}\right)^{2}
$$

Assume that $f(x, y)$ is a continuous function, then $x_{0}$ and $y_{0}$ are the extremums that satisfy the first derivative:

$$
\frac{\partial f}{\partial x}\left(x_{0} ; y_{0}\right)=\frac{\partial f}{\partial y}\left(x_{0} ; y_{0}\right)=0
$$

Afterward, the constant $k^{\prime}$ in Equation (2) can be calculated as:

$$
k^{\prime}=\frac{y_{0}}{\log x_{0}}
$$

Therefore, the general logarithmic function can be determined as follow:

$$
S=\frac{y_{0}}{\log x_{0}} \log I
$$

In this research, some experiments were performed following the "method of limits" [11] by asking the subjects to watch a movie with gradually decreasing the video rate. Thereby, the video rate was deteriorated from the highest to the lowest level within a pre-defined range of $j$ levels. The subtraction of the current video rate level from the highest level was the stimulus intensity. Thus, the stimulus intensity was gradually increased respect for the decrease of the video rate. In the experiment, the duration time between each decrement was about $5 \mathrm{~s}$, thus, the current level of intensity was considered as the reference intensity for a judgment of the next higher intensity level.

The following assumptions need to be subsequently made:

- Data point $P\left(x_{0}, y_{0}\right)$ exists.

- $x_{0}$ is either absolute threshold or just noticeable intensity.

- Let $X_{m}$ be the $m^{\text {th }}$ reference intensity for a judgment of $(m+1)^{\text {th }}$ intensity with $1 \leq m \leq j$.

- Let $X_{\text {threshold }}$ be the stimulus threshold that needs to be determined.

If $x_{0}$ is equal to the absolute threshold, the stimulus threshold is ideally determined as: $X_{\text {threshold }}=x_{0}$.

If $x_{0}$ is recognized as the just noticeable intensity, then, the absolute threshold is need to be determined. Actually, only the constraint of absolute threshold can be determined in this case. Because the constant $k$ in Weber's Law (Equation (1)) is unknown, thus, the next higher intensity cannot be ascertained from the current one. Accordingly, the absolute threshold is derived from the following constraint:

$$
X_{n} \leq X_{\text {threshold }} \leq x_{0}
$$

\section{Evaluation}

In this section, the hypotheses in this research are practically confirmed through some experiments. In the experiments, subjects watched short video clips. Video quality of clips was gradually changed from the highest to the lowest level while 
SC and HR data were being continuously recorded.

\subsection{Experimental Environment}

Figure 4 shows the experimental setup which comprises of a screen, a MIO watch (for measuring heart rate) connected to Mio app on iPhone 6 via Bluetooth and a Grove-GSR sensor [40] connect to Arduino UNO board (for measuring skin conductance). The sampling frequency for HR and SC monitoring were $1 \mathrm{~Hz}$ and $20 \mathrm{~Hz}$, respectively. Note that the sampling frequency of HR was fixed by the device vendor, whereas the one of SC was flexibly changeable.

In order to maximize the reliability of the experiments, the video clips should satisfy some requirements. The video material utilized in these experiments should not be a semantically important content or a salient content. As the result, the influences due to high-level image understanding were eliminated. Furthermore, the duration of watching the video should not be too long in order to avoid the distraction of the subjects. Also, the video rate must have been decreased level by level.

To meet these requirements, a video clip generated from an open source $4 \mathrm{k}$ movie was prepared as follows:

- The utilized video clip had an abstract content; a view of sky at night which slowly moves from the left to the right.

- The original video clip was encoded to Internet Information Services (IIS) smooth streaming [41] -720 CBR (constant bitrate) by using Microsoft Encoder pro 4.0 which allows it to be available with multiple video rates (kbps): 2962, 2056, 1427, 991, 688, 477, 331 and $230 \mathrm{kbps}$. The reason to encode the video with constant bitrate instead of variable bitrate is to ensure that the video clips always keeps the same pre-defined bitrate level. The stimulus intensities were calculated by doing the subtraction of current video rate level from the highest level of $2962 \mathrm{kbps}$. The stimulus intensities were: $906,1535,1971,2274,2485,2631$, and $2732 \mathrm{kbps}$ (Note that by default the video clip initially started from the highest video rate of $2962 \mathrm{kbps}$ and the first decrease started at $2056 \mathrm{kbps}$ ).

- A seamless video clip of 40 seconds which comprises of eight 5-second-chunks
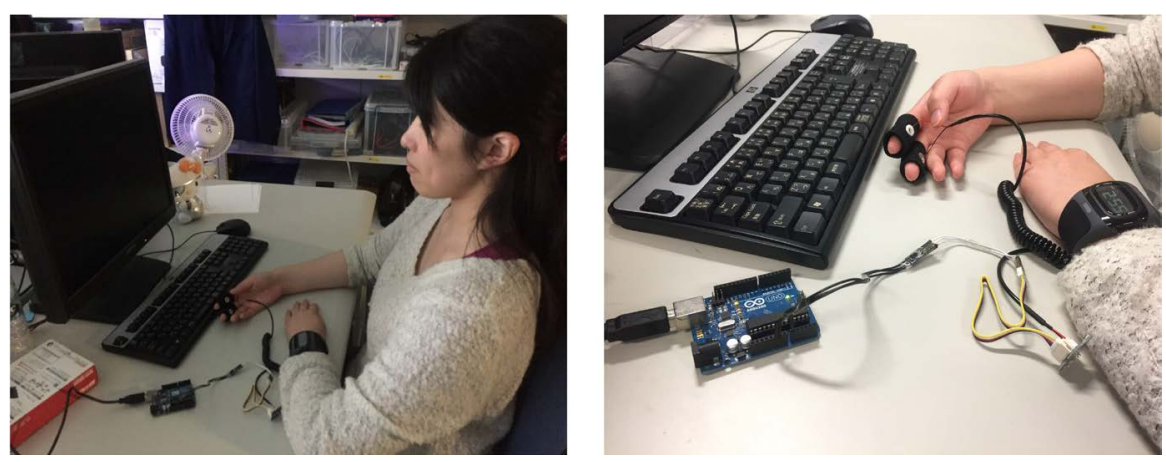

Figure 4. Environmental Setup with MIO watch and Grove-GSR sensor. 
was generated. Each of chunk is respectively available with each video rate mentioned above. Therefore, this video clip contains seven stimulus intensities. In addition, the current stimulus intensity was considered as the reference intensity of the judgment for the next higher intensity.

The video clip was displayed on a 22 -inch screen Samsung SyncMaster $2243 \mathrm{BW}$ with a native resolution of $1680 \times 1050$. The video resolution was 1276 $\times 660$ pixels or $32.4 \times 16.8 \mathrm{~cm}$ and the viewing distance was $67.2 \mathrm{~cm}$ (four times of the video height on the screen) in compliance with the specifications in [42]. Ten subjects (4 females and 6 males in the age group of 20 - 27) participated in the experiment. They were doing the researches not related to this research topic. All subjects had normal or corrected-to-normal vision. The subjects sat in front of the display in a dark and quiet room. They were also asked to leave all their mobile phones or any noise-making devices which could distract them during the experiment. After attaching the measurement devices, the subjects had five minutes at rest to be familiar with the experimental environment that made them achieve the highest comfortable status. When the subjects were ready, they clicked a button on the screen to play the video, whereas both HR and SC recordings were also started. The experiment was repeated two times for each subject.

\subsection{Data Acquisition and Data Transformation}

Skin conductance raw data was originally read from the serial port on the computer using a simple program written in Python. Then, it was exported to CSV format followed by its transformation to "txt" file which is suitable input form for Ledalab-an open source SC data analysis tool [43] [44]. According to [45], there are individual differences in amplitude of SC. In other words, the overall response of a subject is not the same as the one of others. Therefore, it is important to normalize and standardize the raw data collected from the above experiment.

In this research, the skin conductance data was transformed following the two-phase: normalization and standardizations [46]. Firstly, the raw data was normalized based on the Equation (9). Afterward, the data was standardized by calculating the ratio of normalized data and its mean with Equation (10).

$$
\begin{gathered}
\mathrm{SC}_{\text {nor }}=\frac{\mathrm{SC}-\mathrm{SC}_{\min }}{\mathrm{SC}_{\max }-\mathrm{SC}_{\min }} \\
\mathrm{SC}_{\mathrm{sta}}=\frac{\mathrm{SC}_{\text {nor }}}{\mathrm{E} \times\left(\mathrm{SC}_{\text {nor }}\right)}
\end{gathered}
$$

Here, $\mathrm{SC}_{\text {nor }}$ is normalized SC data, $\mathrm{SC}_{\min }$ and $\mathrm{SC}_{\max }$ are the minimum and maximum SC values, respectively. Meanwhile, $\mathrm{SC}_{\text {sta }}$ is standardized SC data, $\mathrm{E} \times\left(\mathrm{SC}_{\mathrm{nor}}\right)$ is the mean of the normalized SC data.

After normalization and standardization, SC data needs to be analyzed to confirm the hypotheses throughout the following criteria:

1) Event-related skin conductance response (ER-SCRs) of each subject. 
2) Regression model which represents the relation between SC and stimulus's level.

Firstly, ER-SCRs presents the responses of subjects for a given stimulus. The number of responses as well as the amplitude of responses play a critical role in investigating how each subject reacts to the stimulus. As mentioned in Section 3, SC data comprises of tonic and phasic components. Thus, to extract ER-SCRs, the SC data is decomposed into its tonic and phasic components by Ledalab tool. The decomposition results in the extraction of un-superposed response components and thus allows for an unbiased quantification of SCR characteristics (e.g., SCR amplitude). The following outputs should be derived from the decomposition: the number of response and SCR amplitude. SC theoretically reacts to a stimulus by skin conductance response and the SCR usually occurs about one to five seconds after stimulus's onset [28] [47]. Therefore, the outputs were obtained within the pre-defined response window (from 1 to 5 seconds).

Secondly, to model the impact of stimuli on skin conductance, the average value of $\mathrm{SC}_{\text {sta }}$ data was calculated within the response window. Therefore, for each trial of experiment, there were totally seven average values according to seven setup-stimulus. By performing the logarithmic approximation, the abovementioned relation was established.

Heart rate raw data was initially stored on Mio app where both the average value and the general trend were clearly shown off. The raw data can be extracted by email as the attached files. The following criteria were used to confirm the hypotheses of this research:

1) The variation of $\mathrm{HR}$ within the pre-defined response window.

2) Logarithmic approximation obtained from the normalized data.

The variation of HR stands for the standard deviation which presents the fluctuation of HR data from the subject's resting HR baseline. Before modeling the relation between HR and stimulus, the raw data must be normalized by applying the following equation:

$$
\mathrm{HR}_{\text {nor }}=\frac{\mathrm{HR}-\mathrm{HR}_{\text {min }}}{\mathrm{HR}_{\text {max }}-\mathrm{HR}_{\text {min }}}
$$

Here, $\mathrm{HR}_{\text {nor }}$ is normalized $\mathrm{HR}$ data, $\mathrm{HR}_{\text {min }}$ and $\mathrm{HR}_{\max }$ are the minimum and maximum HR values, respectively.

\subsection{Numerical Results}

This subsection shows the numerical results of SC and HR measurement followed by analyzing criteria mentioned in Subsection 5.2. Consequently, the hypotheses were practically justified.

Skin conductance data, in general, shows that the amplitudes of responses varies from person to person due to the difference of their skin properties. Table 1 presents an example of the experimental result, which shows the discrete decomposition analysis (DDA) of a subject. DDA is a method to decomposes the 
SC data into the tonic and discrete phasic components. The result implies how the particular subject perceives the stimulus's intensities through the number of significant SCRs and the amplitude of significant SCRs. The stimulus intensities are shown in the first column "Event.Name". Whereas, the number of significant SCRs (ER-SCRs) and the amplitude of significant SCRs are presented in the column of "DDA.nSCR" and "DDA.AmpSum". Data in column "DDA.Latency" presents the response latency of the first significant SCR. Lastly, "DDA.Tonic" column shows the mean tonic activity of decomposed tonic component. The numbers of responses and the amplitudes decrease (sometimes decrease to zero) when stimulus intensity increases. Figure 5 illustrates the amplitude of SCR obtained from each subject. It can be seen that the amplitude varies from subject to subject according to the stimulus intensity increment.

Table 1. NS-SCR analysis in SC data. The results were the output of Discrete Decomposition Analysis (DDA) done by lelalab tool.

\begin{tabular}{ccccc}
\hline Event.Name & DDA.nSCR & DDA.Latency & DDA.AmpSum & DDA.Tonic \\
\hline 906 & 1 & 4.075 & 0.749 & 5.464 \\
1535 & 1 & 3.775 & 0.610 & 5.464 \\
1971 & 1 & 4.375 & 0.431 & 5.464 \\
2274 & 1 & 3.875 & 0.290 & 5.464 \\
2485 & 0 & $\mathrm{NaN}$ & 0 & 5.464 \\
2631 & 1 & 1.575 & 0.310 & 5.464 \\
2732 & 0 & $\mathrm{NaN}$ & 0 & 5.464 \\
\hline
\end{tabular}

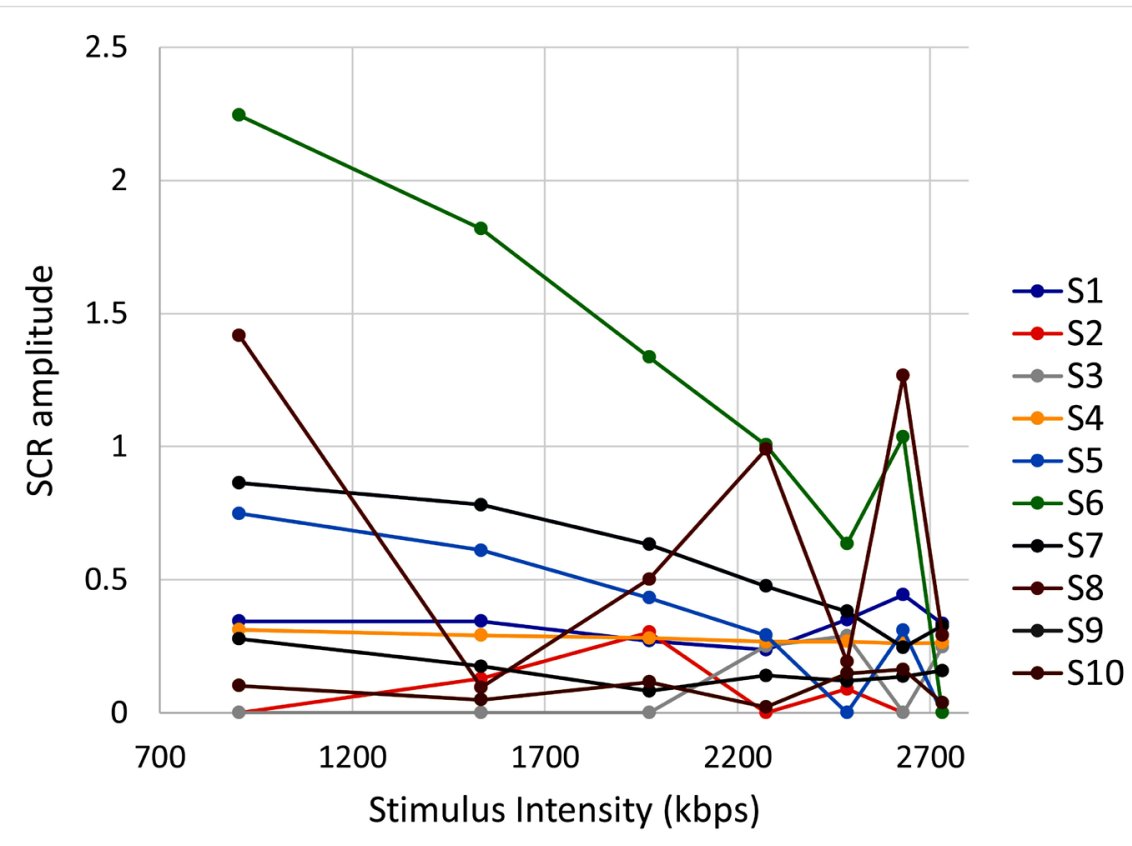

Figure 5. The SCR-amplitude of significant SCR re-convolved from corresponding phasic driver-peaks. 
Heart rate data presents the same implication as SC data when subjects differently perceive the stimuli. As mentioned in Subsection 5.2, the standard deviation as the variation of HR from the resting HR baseline was a crucial criterion. Figure 6 illustrates the standard deviation of HR in each video rate within pre-defined response window. In the figure, each color shows each subject. In general, there is no conspicuous consistency among subjects. Some subjects, e.g. subject 1, 2, 8 and 9, expose high variations when stimulus intensity increases to the values of $1535 \mathrm{kbps}$ and $2274 \mathrm{kbps}$.

After confirming the first hypothesis, the tasks mentioned in Subsection 4.2 need to be accomplished. Initially, the two series of logarithmic nature regression curves of Equation (3) were established by respectively fitting SC data and HR data obtained from all subjects. As the result, each series of either SC data or HR data comprised of ten curves of ten subjects. The accuracy of those logarithmic approximation was represented by correlation of determination denoted by $\mathrm{R}$-squared. The according R-squared of twenties regression curves are shown in Table 2. It is clear to see that SC data produces a better approximation with R-squared of 0.78892 and higher, except subject 3 and 4. Meanwhile, oppositely, $\mathrm{R}$-squared of $\mathrm{HR}$ is just around 0.38341 and lower, except subject 1, 5 and 9. It means that the relation between heart rate and stimulus was not well fitted.

Investigating the existence and determination of data point $P\left(x_{0}, y_{0}\right)$ are the next tasks in this subsection. The functions of regression curves taken from SC data have the following form:

$$
y_{i}=a_{i} \log x+b_{i}
$$

where $i \in\{1,2, \cdots, n\}$ is the index indicating each subject, and $y_{i}$ is the perception level of subject $i$ to the stimulus of SC. $a_{i}$ and $b_{i}$ are constants of those functions. Let $X$ denote $\log X$ in Equation (12) for simplicity.

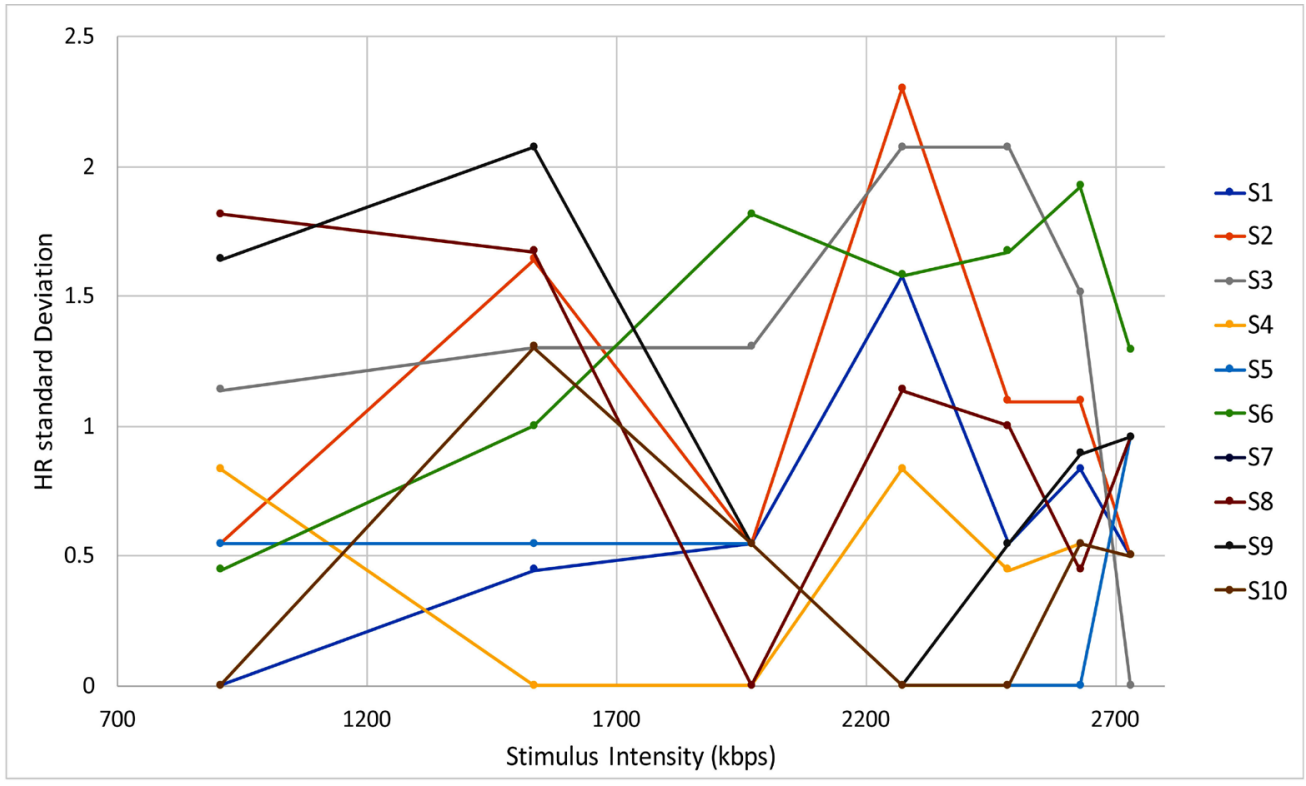

Figure 6. Standard deviation of heart rate data obtained from particular subject. 
As mentioned in Subsection 4.2, to ensure that $x_{0}$ is the absolute threshold or the just noticeable intensity, the data point $P\left(x_{0}, y_{0}\right)$ must be crossed by at least $50 \%$ of regression curves. Figure 7 depicts the regression curves of SC data obtained from 10 subjects. Visually, it is clear that the curves of subject 3 and 4 do not intersect with the rest of curves at the same data point. As the initial prediction, eight curves obtained from the rest of subjects seems to intersect at one data point. Thus, the intensity of this data point is predictably perceived

Table 2. The correlation of determination denoted by R-squared obtained from each subject in both SC data and HR data.

\begin{tabular}{ccc}
\hline Subject & R-squared & R-squared $_{\mathrm{HR}}$ \\
\hline 1 & 0.99084 & 0.83597 \\
2 & 0.89269 & 0.25126 \\
3 & 0.18767 & 0.38341 \\
4 & 0.16698 & 0.36150 \\
5 & 0.87721 & 0.81180 \\
6 & 0.90581 & 0.00350 \\
7 & 0.92398 & 0.58030 \\
8 & 0.91765 & 0.27501 \\
9 & 0.99920 & 0.79140 \\
10 & 0.78892 & 0.21132 \\
\hline
\end{tabular}

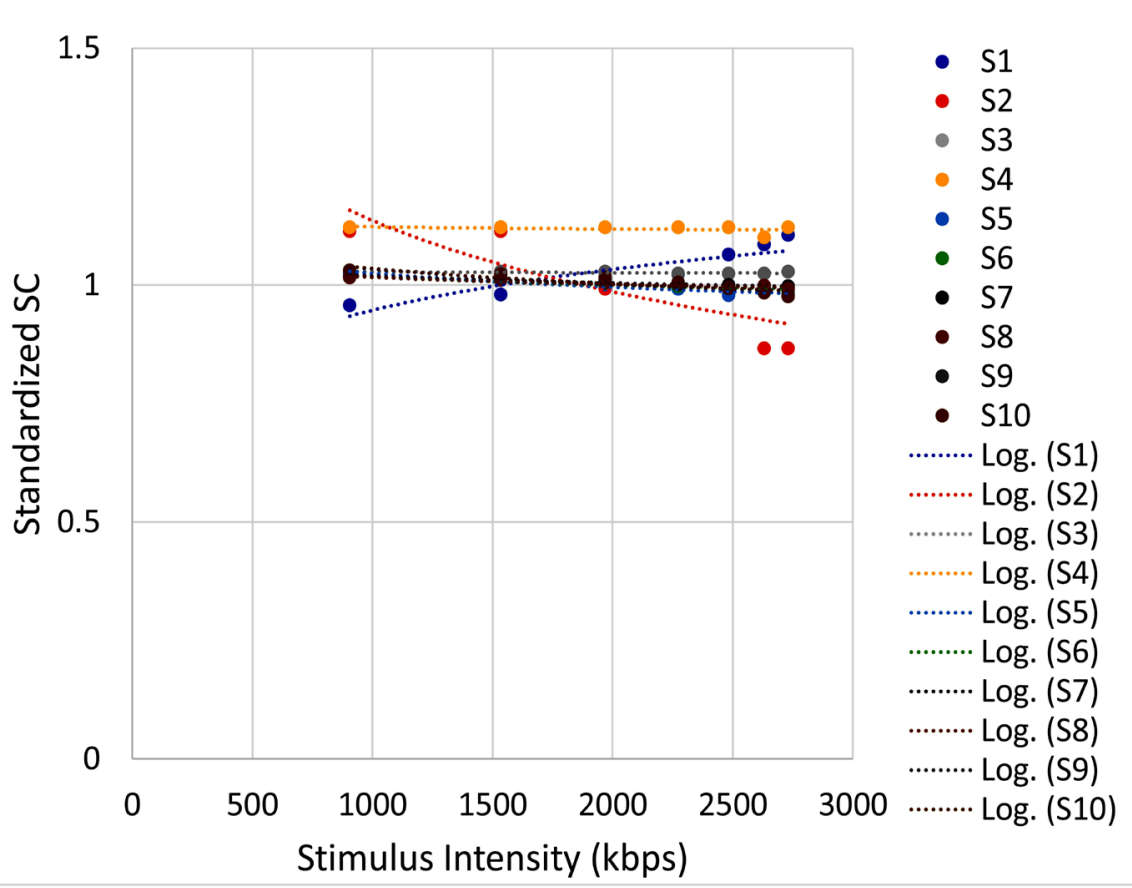

Figure 7. The logarithmic nature regression curves of SC data obtained from 10 subjects. $\log S_{i}$ means the logarithmic nature curves, whereas $S_{i}$ is the data point of each subject. 
by about $80 \%$ of subjects (except subject 3 and 4 ). In other words, it turns out to be a just noticeable intensity. To confirm this, the method of least square in Equation (4) was performed determining the data point $P\left(x_{0}, y_{0}\right)$. The requirement is to find $x_{0}$ and $y_{0}$ satisfying the minimum sum of squared residuals, defined as the square of the difference between $y$ and $y_{i}$.

Then $x_{0}$ and $y_{0}$ are the extremums that respectively satisfies the first derivative of Equation (5). The values of $x_{0}$ and $y_{0}$ were eventually calculated as $2113.62 \mathrm{kbps}$ and $1.0079 \mathrm{kbps}$, respectively. This means that there is the existence of one data point which is crossed by eight regression curves in the investigating range of stimulus intensity, that is to say, from $906 \mathrm{kbps}$ to $2732 \mathrm{kbps}$. This leads to the fact that $x_{0}$ was confirmed as the just noticeable intensity. Therefore, based on the implication in Subsection 3.3, the data point $P\left(x_{0}, y_{0}\right)$ must belong to the general logarithmic curve of Equation (2). Then, from the value of $x_{0}, y_{0}$, the constant $k^{\prime}$ in general logarithmic function is calculated as: $k^{\prime}=\frac{y_{0}}{\log x_{0}}=0.13$.

Consequently, the general logarithmic function which expresses the relation between SC and stimulus intensity will be: $y=0.13 \log x$.

According to the Subsection 4.2, due to the unknown of constant $k$ in Weber's Law (Equation (1)), it is impossible to determine the absolute threshold. Instead of this, the constraint of the absolute threshold is established. Because $x_{0}=2113.62 \mathrm{kbps}$ and being in the range intensity of $(1971,2274)$, thus, the reference intensity is $X_{m}=1971 \mathrm{kbps}$. According to Equation (8), the constraint of the absolute threshold is determined as: $1971 \mathrm{kbps} \leq X_{\text {threshold }} \leq 2113.62 \mathrm{kbps}$.

By subtracting the reference intensity and just noticeable intensity from the highest video rate level of $2962 \mathrm{kbps}$, the video rate threshold $B R_{\text {threshold }}$ is accordingly defined: $848.38 \mathrm{kbps} \leq B R_{\text {threshold }} \leq 991 \mathrm{kbps}$.

For HR data, the same method was expected to perform for determination of either absolute threshold or its constraint. However, according to Table 2, only three regression curves have high accuracy in terms of correlation of determination R-squared. It means that the intensity $x_{0}$ of data point $P\left(x_{0}, y_{0}\right)$ was perceived with the same amplitude of perception by only $30 \%$ of subjects. Thus, the existence of both absolute threshold and just noticeable intensity, that is to say, perceived by at least $50 \%$ of subjects is excluded. Eventually, the only $B R_{\text {threshold }}$ can be derived from SC data. According to [48], HAS player always attempt to maintain a constant gap between the target video rate and the needed bandwidth. Such a constant gap is determined by the following equation:

$$
\operatorname{margin}_{\text {ratio }}=\frac{B W-\operatorname{target} B R}{B W}
$$

where, $B W$ is the available bandwidth, and target $B R$ is the expected video representation (target video rate) of the users. Margin $_{\text {ratio }}$ refers to a conservatism value defined by particular proprietary HAS players. In fact, Microsoft smooth streaming applies a conservatism value of 20\% [48]. 
Accordingly, the required bandwidth $B W$ for $B R_{\text {threshold }}$ is calculated as: $1060.48 \mathrm{kbps} \leq B W \leq 1238.75 \mathrm{kbps}$. On the other hand, according to [15], by using PSQA model, the values of QoS parameters (bandwidth, packet loss, delay and jitter) can be interpreted to MOS, and vice versa. Therefore, this constraint is then converted into optimal MOS constraint: $2.78 \leq \operatorname{MOS}_{\text {threshold }} \leq 2.91$ with the assumption that packet loss, delay, and jitter are negligible.

\section{Discussion}

In this section, it is important to discuss the validation of this optimal constraint of MOS, and thus, a simple experiment was conducted. In this experiment, ten subjects (they are different from the subjects in the experiment in Section 5) were asked to watch a short movie with the duration of 2 minutes, then, to provide their subjective evaluation following the 5-scale MOS. There were two scenarios in this experiment in order to validate the performance of optimal $\mathrm{MOS}_{\text {threshold }}$ in comparison with the fair $\mathrm{MOS}_{\text {threshold }}$. In both scenarios, the movie's video rate was gradually decreased in response to the negatively changing the network QoS parameters (bandwidth, packet loss, delay and jitter), while the estimated MOS was being calculated by PSQA model [15] [49] [17]. When the estimated MOS decreases to $\operatorname{MOS}_{\text {threshold }}$, the control action will be triggered (control action is activated by allocating the higher bandwidth to the user [50]). In this case, the optimal $\operatorname{MOS}_{\text {threshold }}$ was equal to 2.78 which is the lowest value of optimal constraint. The fair $\operatorname{MOS}_{\text {threshold }}$ was equal to 3 which is defined as the fair value in 5-scale MOS [16] [17] [18]. The subjective MOS collected from subjects in the first scenario was compared with the one obtained in the second scenario. The validation criteria were the similarity of subjective MOS and network resource utilization in both scenarios.

To investigate the similarity of subjective MOS, the analysis of variance (ANOVA) approach [51], especially one-way ANOVA which is a technique for comparison means of two or more samples (using $\mathrm{F}$ distribution), was accomplished. The result of ANOVA indicates that the overall subjective MOS which were obtained from all the subjects in both scenarios were equal.

Now resource utilization is in turn considered. Thus, the required bandwidth allocations, which are sufficient for the users to experience the video quality with MOS higher than optimal and fair $\operatorname{MOS}_{\text {threshold }}$, were investigated. As mentioned in Section 5, the required bandwidth for $\mathrm{MOS}_{\text {threshold }}$ of 2.78 was equal to $1060.48 \mathrm{kbps}$. Assume that the values of QoS parameters, except bandwidth, were negligible during the experiments. By using the PSQA model, the required bandwidth for fair $\mathrm{MOS}_{\text {threshold }}$ of 3 was calculated to be equal to $1303.23 \mathrm{kbps}$. The setup of this experiment was the same as those of our previous works in [38], where the link capacity was limited to $5000 \mathrm{kbps}$. Therefore, it is clear to see that in terms of resource utilization, using the optimal $\mathrm{MOS}_{\text {threshold }}$ can save at least $\frac{1303.23-1060.48}{5000} * 100=4.855 \%$ of bandwidth allocation per control compared 
to the fair $\mathrm{MOS}_{\text {threshold }}$. In terms of resource utilization, this result might be modest. However, it will be significantly meaningful for the practical system which is much larger and more complicated than the one in this research.

The validation result shows that the constraint of QoE threshold optimally satisfies the research purpose of QoE management, that is to say, ensuring that QoE is stable at an expected level with minimal network resource usage. Therefore, using biological information produces a prominent result in modeling a logarithmic relation of human perception and stimulus intensity.

However, there are some other issues also need to be discussed. Firstly, the particular regression analysis of either SC data or HR data obtained from each subject did not always offer the expected result with sufficient accuracy. For SC data, the results from some subjects surprisingly produced very low correlation of determination, especially subject 4. According to the result of DDA in Subsection 5.3, subject 4 also did not produce the significant SCRs for most of intensities. Thus, during the experiment, this subject maybe did not concentrate or got some invisible distractions. For HR data, only $30 \%$ of subjects produced high accuracy regression curves leading to its exclusion of this research. As stated in Subsection 5.1, the sampling frequency of $\mathrm{HR}$ was only $1 \mathrm{~Hz}$ and was fixed by the device vendor. In addition, the value of $\mathrm{HR}$ obtained from monitoring device is the average value. Therefore, HR measurement cannot capture significant biological characteristics, resulting in a miserable regression analysis. However, using HR monitoring device can avoid the intrusiveness which was recognized as one of the major limitations of psychophysiology in [9]. In addition, the introduction of wearable device which is capable of measuring both $\mathrm{HR}$ and heart rate variability (HRV) can significantly improve the accuracy in QoE assessment due to the fact that HRV provides a powerful means of observing the interplay between the sympathetic and parasympathetic nervous systems [52].

Human characteristic is another discussion point. In the perceptual process, sensation refers to the initial steps-converting physical features of the environment into electrochemical signals within specialized nerve cells and sending those signals to the brain for processing. Meanwhile, perception refers to the last steps, whereby the initial sensory signals are used to form mental representations of the objects and events in a scene so that they can be recognized. Therefore, the electrical activity of the brain measured by Electroencephalography (EEG) should be taken into account in this research. The combination of EEG associated with Central Nervous System (CNS) and measurement methods associated with Autonomic Nervous System (ANS) will promisingly produce a better result in modeling the relation between perception and stimulus intensity applying in QoE management research.

\section{Conclusion and Future Works}

In this paper, the relation between biological information and stimulus intensity has been modeled as a general logarithmic nature function. Thereby, the optimal 
constraint of QoE threshold has been determined and validated. The results of validation show that by using the optimal threshold, not only the overall QoE is guaranteed to be stable at high level, but also the network resource utilization is improved. The obtained threshold constraint is suitable for only the scenario in this research, but the approach can be applied in more general cases. This research also confirmed the possibility of applying biological information in QoE management. However, the result of this study also does not provide a well-correlation between HR data and stimulus intensity. Therefore, the other signals such as HRV, EEG, ECG should be investigated in the future works.

\section{Conflicts of Interest}

The authors declare no conflicts of interest regarding the publication of this paper.

\section{References}

[1] Move Networks (2008) The Introduction of Adaptive Video Streaming. American Fork.

[2] YouTube for Press. https://www.youtube.com/intl/en-GB/yt/about/press/

[3] IBM (2017) The State of Streaming 2017 Report. Armonk.

[4] Seppänen, J., Varela, M. and Sgora, A. (2014) An Autonomous QoE-Driven Network Management Framework. Journal of Visual Communication and Image Representation, 25, 565-577.

[5] Agboma, F. and Liotta, A. (2012) Quality of Experience Management in Mobile Content Delivery Systems. Telecommunication Systems, 49, 85-98.

https://doi.org/10.1007/s11235-010-9355-6

[6] Menkovski, V., Exarchakos, G., Liotta, A. and Sánchez, A.C. (2010) Quality of Experience Models for Multimedia Streaming. International Journal of Mobile Computing and Multimedia Communication, 2, 1-20.

[7] Mok, R.K.P., Chan, E.W.W. and Chang, R.K.C. (2011) Measuring the Quality of Experience of HTTP Video Streaming. 12 th IFIP/ IEEE International Symposium on Integrated Network Management (IM 2011) and Workshops, Dublin, 23-27 May 2011, 485-492.

[8] Schatz, R., Hoßfeld, T., Janowski, L. and Egger, S. (2013) From Packets to People: Quality of Experience as a New Measurement Challenge. In: Biersack, E., Callegari, C. and Matijasevic, M., Eds., Data Traffic Monitoring and Analysis, Springer, Berlin, Heidelberg, 219-263.

[9] Engelke, U., Darcy, D.P., Mulliken, G.H., Bosse, S., Martini, M.G., Arndt, S., Antons, J.-N., Chan, K.Y., Ramzan, N. and Brunnsträm, K. (2017) Psychophysiology-Based QoE Assessment: A Survey. IEEE Journal of Selected Topics in Signal Processing, 11, 6-21. https://doi.org/10.1109/JSTSP.2016.2609843

[10] Menkovski, V., Exarchakos, G. and Liotta, A. (2011) The Value of Relative Quality in Video Delivery. Journal of Mobile Multimedia, 7, 151-162.

[11] Ghareeb, M. and Viho, C. (2010) Hybrid QoE Assessment Is Well-Suited for Multiple Description Coding Video Streaming in Overlay Networks. Communication Networks and Services Research Conference (CNSR), Montreal, 11-14 May 2010, 327-333. https://doi.org/10.1109/CNSR.2010.15 
[12] Huynh-Thu, Q. and Ghanbari, M. (2012) The Accuracy of PSNR in Predicting Video Quality for Different Video Scenes and Frame Rates. Telecommunication Systems, 49, 35-48. https://doi.org/10.1007/s11235-010-9351-x

[13] Wang, Z., Lu, L.G. and Bovik, A.C (2004) Video Quality Assessment Based on Structural Distortion Measurement. Signal Processing. Image Communication, 19, 121-132. https://doi.org/10.1016/S0923-5965(03)00076-6

[14] Mohamed, S. and Rubino, G. (2002) A Study of Real-Time Packet Video Quality Using Random Neural Networks. IEEE Transactions on Circuits and Systems for Video Technology, 12, 1071-1083. https://doi.org/10.1109/TCSVT.2002.806808

[15] Rubino, G. (2006) Quantifying the Quality of Audio and Video Transmissions over the Internet: The PSQA Approach. In: Barria, J.A., Ed., Communication Networks and Computer Systems. A Tribute to Professor Erol Gelenbe, World Scientific, 235-250. https://doi.org/10.1142/9781860948947_0014

[16] Streijl, R.C., Winkler, S. and Hands, D.S. (2016) Mean Opinion Score (MOS) Revisited: Methods and Applications, Limitations and Alternatives. Multimedia Systems, 22, 213-227. https://doi.org/10.1007/s00530-014-0446-1

[17] Piamrat, K., Ksentini, A., Viho, C. and Bonnin, J.-M. (2008) QoE-Aware Admission Control for Multimedia Applications in IEEE 802.11 Wireless Networks. IEEE 68 th Vehicular Technology Conference, Calgary, 21-24 September 2008, 1-5. https://doi.org/10.1109/VETECF.2008.310

[18] Liotou, E., Tsolkas, D., Passas, N. and Merakos, L. (2015) Quality of Experience Management in Mobile Cellular Networks: Key Issues and Design Challenges. IEEE Communications Magazine, 53, 145-153. https://doi.org/10.1109/MCOM.2015.7158278

[19] Hoßfeld, T., Biedermann, S., Schatz, R., Platzer, A., Egger, S. and Fiedler, M. (2011) The Memory Effect and Its Implications on Web QoE Modeling. 23rd International Teletraffic Congress, San Francisco, 6-9 September 2011, 103-110.

[20] Yamazaki, T., Miyoshi, T., Eguchi, M. and Yamori, K. (2014) Quality of Experience Modeling with Psychological Effect for Interactive Web Services. IEEE Network Operations and Management Symposium, Krakow, 5-9 May 2014, 1-4.

[21] Siris, V.A., Balampekos, K. and Marina, M.K. (2014) Mobile Quality of Experience: Recent Advances and Challenges. IEEE International Conference on Pervasive Computing and Communications Workshops, Budapest, 24-28 March 2014, 425-430.

[22] Pham-Thi, H. and Miyoshi, T. (2016) User-Centric Approach for Bandwidth Allocation Method Based on Quality of Experience. IEICE Transactions on Communications, 99, 1282-1290. https://doi.org/10.1587/transcom.2015EUP0008

[23] Ramzan, N., Palke, S., Cuntz, T., Gibson, R. and Amira, A. (2016) Emotion Recognition by Physiological Signals. IS \& T International Symposium on Electronic Imaging, San Francisco, 14-18 February 2016, 1-6.

[24] Kim, C.J., Park, S., Won, M.J., Whang, M. and Lee, E.C. (2013) Autonomic Nervous System Responses Can Reveal Visual Fatigue Induced by 3D Displays. Sensors, 13, 13054-13062. https://doi.org/10.3390/s131013054

[25] Abadi, M.K., Staiano, J., Cappelletti, A., Zancanaro, M. and Sebe, N. (2013) Multimodal Engagement Classification for Affective Cinema. Humaine Association Conference on Affective Computing and Intelligent Interaction, Geneva, 2-5 September 2013, 411-416.

[26] Scholler, S., Bosse, S., Treder, M.S., Blankertz, B., Curio, G., Muller, K.-R. and Wie- 
gand, T. (2012) Toward a Direct Measure of Video Quality Perception Using EEG. IEEE Transactions on Image Processing, 21, 2619-2629. https://doi.org/10.1109/TIP.2012.2187672

[27] Reichl, P., Egger, S., Schatz, R. and D’Alconzo, A. (2010) The Logarithmic Nature of QoE and the Role of the Weber-Fechner Law in QoE Assessment. IEEE International Conference on Communications, Cape Town, 23-27 May 2010, 1-5.

[28] Boucsein, W. (2012) Electrodermal Activity. Springer Science \& Business Media, Berlin. https://doi.org/10.1007/978-1-4614-1126-0

[29] Iarocci, G. and McDonald, J. (2006) Sensory Integration and the Perceptual Experience of Persons with Autism. Journal of Autism and Developmental Disorders, 36, 77-90. https://doi.org/10.1007/s10803-005-0044-3

[30] Fowles, D.C. (1986) The Eccrine System and Electrodermal Activity. Psychophysiology: Systems, Processes, and Applications, 1, 51-96.

[31] McIntosh, D.N., Miller, L.J., Shyu, V. and Hagerman, R.J. (1999) Sensory-Modulation Disruption, Electrodermal Responses, and Functional Behaviors. Developmental Medicine and Child Neurology, 41, 608-615. https://doi.org/10.1017/S0012162299001267

[32] Benedek, M. and Kaernbach, C. (2010) Decomposition of Skin Conductance Data by Means of Nonnegative Deconvolution. Psychophysiology, 47, 647-658.

[33] Chaudhuri, A. (2011) Fundamentals of Sensory Perception. Oxford University Press, Oxford.

[34] Yantis, S. (2013) Sensation and Perception. Palgrave Macmillan, London.

[35] Colman, A.M. (2015) A Dictionary of Psychology. Oxford University Press, Oxford.

[36] Gescheider, G.A. (2013) Psychophysics: The Fundamentals. Psychology Press, Hove.

[37] Seufert, M., Egger, S., Slanina, M., Zinner, T., Hossfeld, T. and Tran-Gia, P. (2015) A Survey on Quality of Experience of HTTP Adaptive Streaming. IEEE Communications Surveys \& Tutorials, 17, 469-492.

https://doi.org/10.1109/COMST.2014.2360940

[38] Phan-Xuan, T. and Kamioka, E. (2017) Accurate Available Bandwidth Allocation in HTTP Adaptive Streaming. International Journal of Computer Networks and Communications, 9, 83-94. https://doi.org/10.5121/ijcnc.2017.9507

[39] Cermak, G., Pinson, M. and Wolf, S. (2011) The Relationship among Video Quality, Screen Resolution, and Bit Rate. IEEE Transactions on Broadcasting, 57, 258-262. https://doi.org/10.1109/TBC.2011.2121650

[40] SeedStudio, GSR Sensor. http://wiki.seeedstudio.com/Grove-GSR_Sensor/

[41] Microsoft, IIS Smooth Streaming. https://www.iis.net/downloads/microsoft/smooth-streaming

[42] Recommendation ITU-R BT (2002) Methodology for the Subjective Assessment of the Quality of Television Pictures.

[43] Karenbach, C. (2005) Ledalab-A Software Package for the Analysis of Phasic Electrodermal Activity. Internal Report, Allgemeine Psychologie, Institut für Psychologie, Institut ftir Psychologie, Tech. Rep.

[44] Benedek, M. (2016) Analysis of EDA Using Ledalab.

[45] Braithwaite, J.J., Watson, D.G., Jones, R. and Rowe, M. (2013) A Guide for Analysing Electrodermal Activity (EDA) \& Skin Conductance Responses (SCRS) for Psychological Experiments. Psychophysiology, 49, 1017-1034. 
[46] Braithwaite, J.J. and Watson, D.G. (2015) Issues Surrounding the Normalization and Standardisation of Skin Conductance Responses (SCRS).

[47] Levinson, D.F. and Edelberg, R. (1985) Scoring Criteria for Response Latency and Habituation in Electrodermal Research: A Critique. Psychophysiology, 22, 417-426. https://doi.org/10.1111/j.1469-8986.1985.tb01626.x

[48] Guguen, C.T., Le Bolzer, F. and Houdaille, R. (2017) Improving User Experience When HTTP Adaptive Streaming Clients Compete for Bandwidth. SMPTE Motion Imaging Journal, 126, 28-34. https://doi.org/10.5594/JMI.2016.2632279

[49] Piamrat, K., Viho, C., Bonnin, J.-M. and Ksentini, A. (2009) Quality of Experience Measurements for Video Streaming over Wireless Networks. 6th International Conference on Information Technology: New Generations, Las Vegas, 27-29 April 2009, 1184-1189.

[50] Houdaille, R. and Gouache, S. (2012) Shaping HTTP Adaptive Streams for a Better User Experience. Proceedings of the 3rd Multimedia Systems Conference, Chapel Hill, 22-24 February 2012, 1-9. https://doi.org/10.1145/2155555.2155557

[51] Morrison, D.F. (2005) Multivariate Analysis of Variance.

[52] Acharya, U.R., Joseph, K.P., Kannathal, N., Lim, C.M. and Suri, J.S. (2006) Heart Rate Variability: A Review. Medical and Biological Engineering and Computing, 44, 1031-1051. 